\title{
Thermodynamics of alkali alkanoates VII. Heat capacity and thermodynamic functions of potassium acetate from 4 to $585 K^{a}$
}

\author{
STANLEY P. NGEYI, IMRANA MALIK," \\ Department of Chemistry, Madonna College, \\ Livonia, MI 48150, U.S.A. \\ and EDGAR F. WESTRUM, Jr. \\ Department of Chemistry, University of Michigan, \\ Ann Arbor, MI 48109, U.S.A.
}

(Received 31 October 1989)

\begin{abstract}
The heat capacity of potassium acetate was measured by equilibrium adiabatic calorimetry from near $4 \mathrm{~K}$ up to $350 \mathrm{~K}$ and by d.s.c. from $325 \mathrm{~K}$ through melting (solid to isotropic liquid) to $585 \mathrm{~K}$. No transition or anomaly was observed below $300 \mathrm{~K}$ in the heat-capacity curve but four anomalies were observed at super-ambient temperatures including a broad and diffuse hump extending from 300 to $375 \mathrm{~K}$. A bifurcated peak was observed between 375 and $436.5 \mathrm{~K}$ with peak temperatures at 411 and $425 \mathrm{~K}$ and with enthalpy increments $\left(\Delta_{\mathrm{trs}} H_{\mathrm{m}}^{\circ}\right)$ of 41.7 and $22.8 R \cdot \mathrm{K}$ and entropy increments $\left(\Delta_{\mathrm{trs}} S_{\mathrm{m}}^{\circ}\right)$ of $0.097 R$ and $0.05 R$, respectively. A peak believed to be of first order (solid-to-solid transformation) was observed at $474 \mathrm{~K}$ having $\Delta_{\mathrm{trs}} H_{\mathrm{m}}^{\circ}$ and $\Delta_{\mathrm{trs}} S_{\mathrm{m}}^{\circ}$ of $194 R \cdot \mathrm{K}$ and $0.407 R$. Finally, a solid-to-(isotropic) liquid transition occurred at $582 \mathrm{~K}$ having $\Delta_{\mathrm{trs}} H_{\mathrm{m}}^{\circ}$ and $\Delta_{\mathrm{trs}} S_{\mathrm{m}}^{\circ}$ of $920 R \cdot \mathrm{K}$ and $1.58 R$.
\end{abstract}

\section{Introduction}

The thermophysical properties of potassium $n$-butanoate (K4C) were measured and established earlier. ${ }^{(1)}$ Two close but separated solid-to-solid transformations were observed between 100 and $200 \mathrm{~K}$ thought at first to be overlapping by earlier d.s.c. studies. $^{(2)}$

In this study we examine the thermophysics of potassium acetate $(\mathrm{K} 2 \mathrm{C})$. We considered the study of this compound to be very crucial in as much as (a) it is the first member of the even series of potassium $n$-alkanoates, (b) the odd-even effect seems to play a role in the thermodynamics of compounds with hydrocarbon tails, ${ }^{(1,3,4)}$ and (c) it has been asserted that the lowest members always behave differently in that the increments per $\mathrm{CH}_{2}$ groups are irregular. ${ }^{(1)}$

${ }^{a}$ The first paper in this series is reference 5 .

${ }^{b}$ Work done while a student at the College of Wooster. 


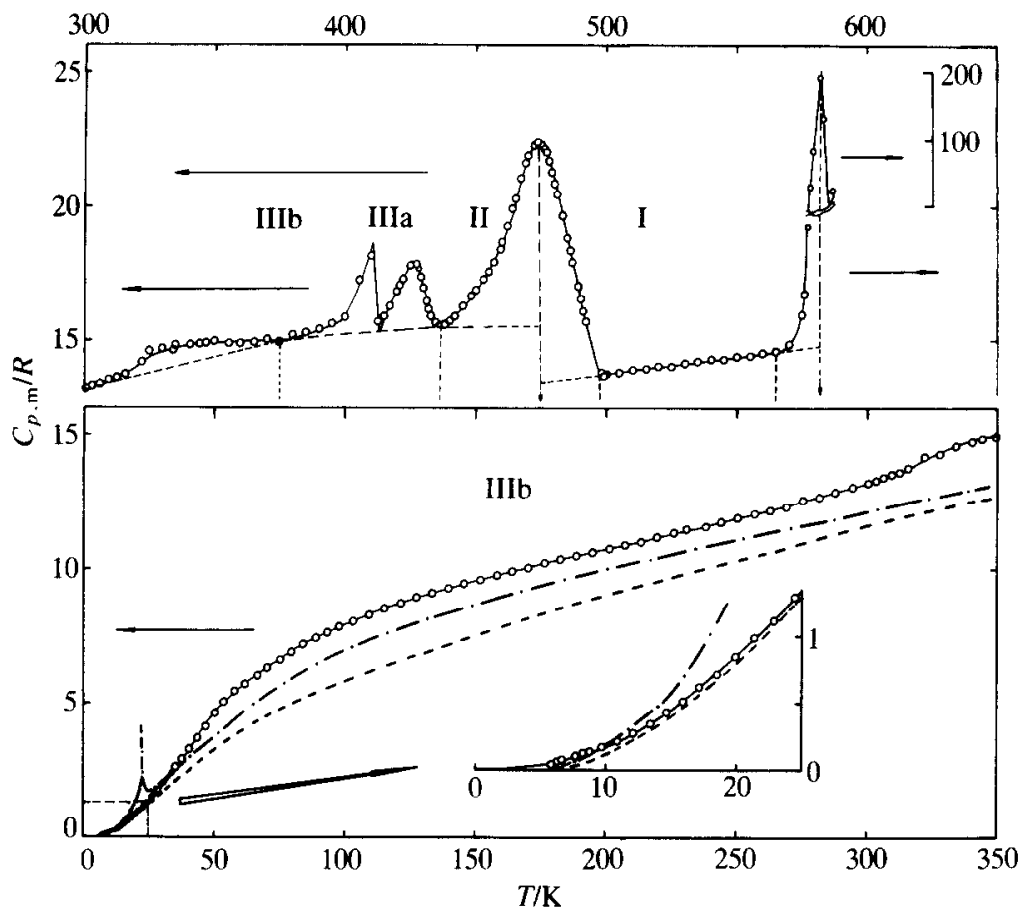

FIGURE 1. The heat-capacity curves of $O$, potassium acetate; $-\cdot-$, , sodium acetate; - - - , lithium acetate. The $21 \mathrm{~K}$ transition of sodium acetate ${ }^{(5)}$ is noted. Designation IIIB refers only to potassium acetate.

The sample does not exhibit a mesomorphic phase. ${ }^{(1)}$ Equilibrium adiabatic calorimetry confirmed the existence of the broad hump also observed by d.s.c. Good agreement between the two methods was obtained over the common temperature range of measurement. The heat-capacity morphology for this sample from near $4 \mathrm{~K}$ through melting is shown in figure 1 . For comparison purposes the heat capacities of sodium and lithium acetates are also shown in this figure. The enthalpies and entropies of the hump and bifurcated peaks not considered to be of first order are also shown in tabular form.

The samples used in both types of measurements were from the same batch. No studies were made on premelted samples.

\section{Experimental}

The potassium acetate used in this sample was prepared by the method described previously. ${ }^{(1,5)}$ The purity was determined by using a DSC-4 purity software with the Dupont Thermal Analysis Data Station (TADS) program ${ }^{(6)}$ which uses the van't Hoff equation as a basis for the analysis. ${ }^{(6,7)}$ The purity of this compound was determined as $\mathbf{9 9 . 9 5}$ moles per cent and considered satisfactory for thermodynamic studies. 
For d.s.c. measurements, the sample was removed from the vacuum line, ${ }^{(8)}$ sealed into the sample pans by crimping, and then placed in the sample compartment of the d.s.c. over which a constant flow of inert gas was maintained to avoid sample oxidation before melting. The d.s.c. used in this study was a computerized DSC-4 version from Perkin-Elmer with adjustment System-4 micro-processor, a computer deck attached to a cathode ray tube, and a computer-controlled chart recorder. The d.s.c. proper also contained an inert atmosphere cover which made it possible to study the hygroscopic samples. Heat-capacity measurements were made using the "Specific Heat" software ${ }^{(9)}$ which is capable of providing $C_{p}(T)$ values at various temperatures. The latter feature made it possible to delineate a true heat-capacity morphology-rather than a d.s.c. trace as with most other d.s.c. instruments-and hence was able to provide the appropriate integrals for thermodynamic properties.

The remaining salt was loaded into a gold-plated oxygen-free high-conductivity copper calorimeter (laboratory designation W-62) placed in the Mark X cryostat $^{(8)}$ provided with computer-assisted calorimetric data acquisition, logging, and programming.

After evacuation of the calorimeter, the sample was loaded and dry helium gas at a pressure of $6.2 \mathrm{kPa}$ was added to promote thermal equilibrium during data acquisition. Leaks in the calorimeter were checked by a helium detector connected to the vacuum line. The mass of the potassium sample was $22.1246 \mathrm{~g}(\cong 0.225436 \mathrm{~mol})$.

Buoyancy corrections were calculated for all masses using a density of $0.941 \mathrm{~g} \cdot \mathrm{cm}^{-3}$ estimated from other members of the homologous series.

The thermal history of the sample is here presented:

$$
\begin{aligned}
& 298 \mathrm{~K} \longrightarrow 54 \mathrm{~K} \underset{10 \mathrm{~h}}{\stackrel{\text { SeriesI }}{\rightarrow}} 161 \mathrm{~K} \underset{11 \mathrm{~h}}{\stackrel{\text { SeriesII }}{\rightarrow}} 315 \mathrm{~K} \underset{3 \mathrm{~h}}{\longrightarrow} 283 \mathrm{~K} \underset{5 \mathrm{~h}}{\stackrel{\text { SericsIIII }}{\rightarrow}} 349.5 \mathrm{~K} \\
& \underset{10 \mathrm{~h}}{\longrightarrow} 5 \mathrm{~K} \stackrel{\text { SerieslV }}{\underset{9 \mathrm{~h}}{\longrightarrow}} 69 \mathrm{~K} .
\end{aligned}
$$

\section{Results and discussion}

The experimental heat capacities are given in table 1 for both d.s.c. and adiabatic calorimetric determinations and are plotted in figure 1. The heat capacities were fitted to a series of orthogonal polynomials in regions where no heat capacity anomalies existed. Integration of these polynomials gave the thermodynamic properties listed in table 2.

By drawing the appropriate lattice heat-capacity curves in the transition regions, excess enthalpies and entropies associated with each transition were calculated and are provided in table 3.

Although potassium acetate has been widely studied ${ }^{(10)}$ by various thermal methods, such as thermal analysis, differential thermal analysis, dilatometry, and visual-polythermal methods, only a few authors ${ }^{(11.12)}$ report excess heat capacity, presumably related to the hump observed by us between 300 and $375 \mathrm{~K}$ with $\Delta_{\mathrm{trs}} H_{\mathrm{m}}^{\circ}$ of about $30.1 R \cdot \mathrm{K}$ and a $\Delta_{\mathrm{trs}} S_{\mathrm{m}}^{\circ}=0.08 R$. For the bifurcated peaks between 375 and $436 \mathrm{~K}$, the total $\Delta_{\mathrm{III}}^{\mathrm{II}} H_{\mathrm{m}}^{\circ}=64.5 R \cdot \mathrm{K}$ and $\Delta_{\mathrm{III}}^{\mathrm{II}} S_{\mathrm{m}}^{\circ}=0.147 R$. These values are in excellent 
TABLE 1. Experimental heat capacity of potassium acetate $\left(\mathrm{KC}_{2} \mathrm{H}_{3} \mathrm{O}_{2}\right)\left(R=8.1345 \mathrm{~J} \cdot \mathrm{K}^{-1} \cdot \mathrm{mol}^{-1}\right)$

\begin{tabular}{|c|c|c|c|c|c|c|c|}
\hline$T / \mathbf{K}$ & $C_{p, \mathrm{~m}} / R$ & $T / \mathbf{K}$ & $C_{p, \mathrm{~m}} / R$ & $T / \mathbf{K}$ & $C_{p, \mathrm{~m}} / R$ & $T / \mathbf{K}$ & $C_{p, \mathbf{m}} / R$ \\
\hline \multicolumn{2}{|c|}{ Series I } & \multicolumn{2}{|c|}{ Series III } & DSC & Data & 465 & 20.27 \\
\hline 54.67 & 5.681 & 286.49 & 12.835 & 325 & 14.64 & 467 & 21.01 \\
\hline 57.10 & 5.401 & 294.71 & 13.059 & 330 & 14.71 & 469 & 21.59 \\
\hline 61.72 & 5.704 & 303.45 & 13.328 & 335 & 14.85 & 470 & 21.91 \\
\hline 66.15 & 6.028 & 309.62 & 13.545 & 340 & 14.89 & 472 & 22.26 \\
\hline 70.61 & 6.338 & 313.80 & 13.765 & 345 & 14.85 & 474 & 22.36 \\
\hline 75.11 & 6.634 & 321.96 & 14.221 & 350 & 14.98 & 475 & 22.23 \\
\hline 79.63 & 6.920 & 328.02 & 14.308 & 355 & 14.84 & 476 & 22.20 \\
\hline 84.18 & 7.212 & 334.10 & 14.631 & 360 & 14.88 & 477 & 21.96 \\
\hline 88.76 & 7.467 & 340.27 & 14.780 & 365 & 14.95 & 479 & 21.28 \\
\hline 93.37 & 7.658 & 346.46 & 14.883 & 370 & 15.05 & 480 & 20.88 \\
\hline 98.00 & 7.844 & \multicolumn{2}{|c|}{ Series IV } & 375 & 14.95 & 481 & 20.45 \\
\hline 103.33 & 8.063 & 5.80 & 0.053 & 380 & 15.24 & 483 & 19.66 \\
\hline 109.37 & 8.304 & 6.19 & 0.069 & 385 & 15.29 & 485 & 18.84 \\
\hline 115.41 & 8.521 & 6.55 & 0.085 & 390 & 15.41 & 487 & 17.89 \\
\hline 121.48 & 8.722 & 6.77 & 0.131 & 395 & 15.61 & 489 & 17.01 \\
\hline 127.55 & 8.950 & 7.02 & 0.089 & 400 & 15.84 & 490 & 16.54 \\
\hline 133.66 & 9.122 & 7.69 & 0.107 & 405 & 17.23 & 491 & 16.06 \\
\hline 139.77 & 9.271 & 8.21 & 0.131 & 410 & 18.12 & 492 & 15.69 \\
\hline 145.89 & 9.465 & 8.76 & 0.138 & 415 & 17.15 & 498 & 13.78 \\
\hline 152.02 & 9.599 & 9.68 & 0.170 & 417 & 16.27 & 499 & 13.68 \\
\hline 158.16 & 9.751 & 10.91 & 0.220 & 420 & 16.83 & 500 & 13.73 \\
\hline \multicolumn{2}{|c|}{ Series II } & 12.13 & 0.276 & 421 & 17.04 & 505 & 13.81 \\
\hline 164.25 & 9.922 & 13.37 & 0.344 & 425 & 17.81 & 510 & 13.92 \\
\hline 170.35 & 10.058 & 14.62 & 0.430 & 427 & 17.86 & 515 & 13.94 \\
\hline 176.52 & 10.244 & 15.89 & 0.518 & 428 & 17.68 & 520 & 14.02 \\
\hline 182.67 & 10.369 & 17.17 & 0.620 & 429 & 17.36 & 525 & 14.02 \\
\hline 188.83 & 10.492 & 18.48 & 0.721 & 430 & 16.94 & 530 & 14.16 \\
\hline 195.01 & 10.622 & 19.89 & 0.849 & 431 & 16.46 & 535 & 14.18 \\
\hline 201.18 & 10.757 & 21.38 & 0.991 & 432 & 16.17 & 540 & 14.29 \\
\hline 207.35 & 10.904 & 22.89 & 1.133 & 433 & 15.90 & 545 & 14.31 \\
\hline 213.52 & 11.024 & 24.41 & 1.294 & 435 & 15.64 & 550 & 14.39 \\
\hline 219.69 & 11.182 & 26.32 & 1.505 & 436 & 15.56 & 555 & 14.42 \\
\hline 225.86 & 11.353 & 27.48 & 1.623 & 437 & 15.53 & 560 & 14.53 \\
\hline 232.04 & 11.511 & 28.67 & 1.777 & 438 & 15.56 & 565 & 14.58 \\
\hline 238.22 & 11.573 & 30.96 & 2.048 & 439 & 15.64 & 570 & 14.85 \\
\hline 244.41 & 11.788 & 33.18 & 2.339 & 440 & 15.72 & 575 & 15.91 \\
\hline 250.59 & 11.923 & 35.44 & 2.631 & 442 & 15.90 & 576 & 16.72 \\
\hline 256.77 & 12.074 & 37.74 & 2.922 & 445 & 16.27 & 577 & 19.21 \\
\hline 262.96 & 12.198 & 40.53 & 3.290 & 448 & 16.64 & 578 & 29.03 \\
\hline 269.14 & 12.355 & 43.80 & 3.700 & 450 & 16.88 & 579 & 83.22 \\
\hline 275.32 & 12.579 & 47.08 & 4.138 & 453 & 17.25 & 580 & 187.4 \\
\hline 281.51 & 12.703 & 50.41 & 4.633 & 455 & 17.52 & 581 & 191.6 \\
\hline 287.70 & 12.861 & 53.77 & 5.043 & 457 & 17.89 & 582 & 192.4 \\
\hline 293.87 & 13.095 & 57.64 & 5.449 & 459 & 18.36 & 583 & 130.2 \\
\hline 305.05 & 13.210 & 62.04 & 5.723 & 460 & 18.63 & 584 & 37.36 \\
\hline 306.23 & 13.419 & 66.48 & 6.042 & 462 & 19.24 & 585 & 21.83 \\
\hline 312.40 & 13.609 & & & 464 & 19.90 & 587 & 24.71 \\
\hline
\end{tabular}

accord with the d.s.c. $422 \mathrm{~K}$ (onset) transition values of Ferloni et al. ${ }^{(2)}$ $(50 R \cdot \mathrm{K}, 0.12 R)$. From crystallographic data, ${ }^{(13)} \Delta_{\mathrm{III}}^{\mathrm{II}} V_{\mathrm{m}}$ is about 2.1 per cent and agrees acceptably with the $\Delta_{\mathrm{trs}} H_{\mathrm{m}}^{\circ}$ value of Jenkins and O'Brien. ${ }^{(14)}$ The presumed association is from one monoclinic phase to another with an eight-fold enhancement 
TABLE 2. Smoothed thermodynamic functions at selected temperatures for potassium acetate $\left(\mathrm{KC}_{2} \mathrm{H}_{3} \mathrm{O}_{2}\right)\left(R=8.3144 \mathrm{~J} \cdot \mathrm{K}^{-1} \cdot \mathrm{mol}^{-1}\right)$

\begin{tabular}{|c|c|c|c|c|}
\hline$T / \mathrm{K}$ & $C_{p, \mathrm{~m}} / R$ & $\Delta_{0}^{T} S_{\mathrm{m}}^{\circ} / R$ & $\Delta_{0}^{T} H_{\mathrm{m}}^{\circ} /(R \cdot \mathbf{K})$ & $\Phi_{\mathrm{m}}^{\circ} / R$ \\
\hline 0 & (0) & $(0)$ & $(0)$ & $(0)$ \\
\hline 5 & $(0.040)$ & $(0.013)$ & $(0.050)$ & $(-0.003)$ \\
\hline 10 & 0.180 & 0.143 & 0.664 & 0.077 \\
\hline 15 & 0.453 & 0.262 & 2.183 & 0.116 \\
\hline 20 & 0.860 & 0.446 & 5.482 & 0.175 \\
\hline 25 & 1.355 & 0.690 & 10.927 & 0.253 \\
\hline 30 & 1.934 & 0.987 & 19.115 & 0.350 \\
\hline 35 & 2.571 & 1.332 & 30.363 & 0.464 \\
\hline 40 & 3.220 & 1.718 & 44.841 & 0.597 \\
\hline 45 & 3.853 & 2.134 & 62.52 & 0.745 \\
\hline 50 & 4.546 & 2.576 & 83.56 & 0.905 \\
\hline 60 & 5.551 & 3.496 & 134.15 & 1.260 \\
\hline 70 & 6.311 & 4.411 & 193.63 & 1.645 \\
\hline 80 & 6.929 & 5.295 & 259.91 & 2.046 \\
\hline 100 & 7.938 & 6.955 & 409.05 & 2.865 \\
\hline 120 & 8.685 & 8.472 & 575.7 & 3.675 \\
\hline 140 & 9.289 & 9.857 & 755.6 & 4.460 \\
\hline 160 & 9.818 & 11.133 & 946.7 & 5.216 \\
\hline 180 & 10.283 & 12.316 & 1147.8 & 5.939 \\
\hline 200 & 10.734 & 13.423 & 1358.0 & 6.633 \\
\hline 220 & 11.202 & 14.468 & 1577.3 & 7.298 \\
\hline 240 & 11.669 & 15.46 & 1806.0 & 7.935 \\
\hline 260 & 12.143 & 16.41 & 2044.1 & 8.548 \\
\hline 280 & 12.657 & 17.33 & 2292.0 & 9.144 \\
\hline 298.15 & 13.155 & 18.14 & 2526.2 & 9.667 \\
\hline 300 & 13.210 & 18.22 & 2550.6 & 9.718 \\
\hline \multirow[t]{2}{*}{325} & 13.2 & $(19.32)$ & $(2892.4)$ & $(10.42)$ \\
\hline & {$[13.2]$} & [19.30] & [2887.5] & {$[10.42]$} \\
\hline \multirow[t]{2}{*}{350} & $(15.0)$ & $(20.42)$ & $(3260.9)$ & $(11.10)$ \\
\hline & [14.3] & {$[20.35]$} & [3238.1] & {$[11.09]$} \\
\hline \multicolumn{5}{|c|}{ Phase II } \\
\hline \multirow[t]{2}{*}{$375^{a}$} & 15.0 & 21.45 & 3633.8 & 11.760 \\
\hline & {$[15.0]$} & {$[21.37]$} & {$[3603.7]$} & {$[11.760]$} \\
\hline \multirow[t]{2}{*}{$411^{b}$} & $(18.7)$ & $(22.84)$ & $(4179.2)$ & $(12.67)$ \\
\hline & [15.3] & {$[22.76]$} & [4149.1] & {$[12.66]$} \\
\hline \multicolumn{5}{|c|}{ Phase IIIa } \\
\hline \multirow[t]{2}{*}{$411^{b}$} & $(18.7)$ & $(22.94)$ & (4220.9) & $(12.67)$ \\
\hline & {$[15.3]$} & {$[22.76]$} & [4149.1] & {$[12.66]$} \\
\hline \multirow[t]{2}{*}{$417.5^{a}$} & (16.3) & $(23.17)$ & $(4320.4)$ & $(12.82)$ \\
\hline & {$[15.3]$} & [22.99] & [4248.6] & {$[12.81]$} \\
\hline \multirow[t]{2}{*}{$425^{b}$} & $(17.8)$ & (23.44) & $(4435.5)$ & $(13.00)$ \\
\hline & {$[15.4]$} & [23.26] & {$[4363.7]$} & {$[12.99]$} \\
\hline \multicolumn{5}{|c|}{ Phase II } \\
\hline \multirow[t]{2}{*}{$425^{b}$} & $(17.08)$ & $(23.49)$ & $(4458.3)$ & $(13.00)$ \\
\hline & {$[15.4]$} & {$[23.26]$} & {$[4363.7]$} & [12.99] \\
\hline \multirow[t]{2}{*}{$436.5^{a}$} & 15.5 & $(23.90)$ & (4635.1) & $(13.28)$ \\
\hline & {$[15.5]$} & [23.67] & [4540.5] & {$[13.27]$} \\
\hline \multirow[t]{2}{*}{$474^{b}$} & (22.5) & (25.23) & $(5214.5)$ & $(14.23)$ \\
\hline & [15.5] & {$[25.00]$} & [5119.9] & {$[14.20]$} \\
\hline
\end{tabular}


TABLE 2 -continued

\begin{tabular}{ccccc}
\hline$T / \mathrm{K}$ & $C_{p, \mathrm{~m}} / R$ & $\Delta_{0}^{T} S_{\mathrm{m}}^{\circ} / R$ & $\Delta_{0}^{r} H_{\mathrm{m}}^{\circ} /(R \cdot \mathrm{K})$ & $\Phi_{\mathrm{m}}^{\circ} / R$ \\
\hline & & Phase I & & \\
$474^{b}$ & $(22.5)$ & $(25.64)$ & $(5408.4)$ & $(14.23)$ \\
& {$[15.5]$} & {$[25.00]$} & {$[5119.9]$} & {$[14.20]$} \\
$497.5^{a}$ & 13.7 & 26.29 & 5727.4 & 14.78 \\
& {$[13.7]$} & {$[25.65]$} & {$[5438.9]$} & {$[14.72]$} \\
500 & 13.8 & 26.39 & 5761.7 & 14.87 \\
510 & 13.9 & 26.69 & 5899.8 & 15.12 \\
520 & 14.0 & 26.99 & 6039.3 & 15.38 \\
530 & 14.1 & 27.29 & 6180.1 & 15.63 \\
540 & 14.3 & 27.59 & 6322.1 & 15.88 \\
550 & 14.4 & 27.89 & 6465.1 & 16.14 \\
560 & 14.5 & 28.19 & 6609.4 & 16.39 \\
$565^{a}$ & 14.6 & 28.49 & 6682.0 & 16.66 \\
& {$[14.6]$} & {$[27.85]$} & {$[6393.5]$} & {$[16.53]$} \\
$582^{b}$ & $\approx 200$ & $(28.92)$ & $(6931.5)$ & $(17.01)$ \\
& {$[14.8]$} & {$[28.28]$} & {$[6643.0]$} & {$[16.87]$} \\
& & & \\
$582^{b}$ & $\approx 200$ & Isotropic liquid & & $(17.01)$ \\
$585^{a}$ & {$[14.8]$} & {$[30.50)$} & $(7851.2)$ & {$[16.87]$} \\
& 21.8 & {$[28.28]$} & {$[6643.0]$} & 17.07 \\
& {$[21.8)$} & {$[28.38]$} & 7915.7 & {$[6707.5]$} \\
\hline
\end{tabular}

${ }^{a}$ These are the (minimum) points on the heat-capacity curve for a given transition or pair of transitions from which extrapolation of the "lattice" heat capacity was made for the calculation of transition (excess) values of the thermodynamic functions.

${ }^{b}$ The values of the thermodynamics of transition are given at the transition temperatures on the assumption that the transitions are strictly isothermal at these temperatures.

of the unit cell volume. The II-to-I solid-to-solid transition is at $474 \mathrm{~K}$ and fusion is at $582 \mathrm{~K}$. When account is taken of the differences between the usual practice of listing d.s.c. transitions by the first deviation from the "lattice" curve on heating, the II-to-I transition at $474 \mathrm{~K} ;{ }^{(12-14)}$ the bifurcated peaks between 375 and $436 \mathrm{~K} ;{ }^{(12)}$

TABLE 3. Transitional thermodynamic properties of potassium acetate $\left(R=8.3145 \mathrm{~J} \cdot \mathrm{K}^{-1} \cdot \mathrm{mol}^{-1}\right)$

\begin{tabular}{lccc}
\hline \multicolumn{1}{c}{ Transition (phases) } & $T_{\mathrm{trs}} / \mathrm{K}^{b}$ & $\Delta_{\mathrm{trs}} H_{\mathrm{m}} /(R \cdot \mathrm{K})^{a}$ & $\Delta_{\mathrm{trs}} S_{\mathrm{m}} / R$ \\
\hline Hump & 300 to $375^{c}$ & $27.6 \pm 0.3$ & $0.080 \pm 0.001$ \\
IIIb-to-IIIa $^{d}$ & $\approx 411$ & $41.7 \pm 0.5$ & $0.097 \pm 0.001$ \\
IIIa-to-II & $\approx 425$ & $22.8 \pm 0.1$ & $0.050 \pm 0.001$ \\
II-to-I & $\approx 474$ & $194 \pm 2$ & $0.407 \pm 0.009$ \\
I-to-Isotropic liquid & $\approx 582$ & $920 \pm 9$ & $1.58 \pm 0.02$ \\
$\quad$ (i.e. melting) & & & \\
\hline
\end{tabular}

${ }^{a}$ By d.s.c. The uncertainties are estimated standard deviations.

${ }^{b}$ These are peak temperatures.

'A broad and diffuse transformation extending from approximately 300 to $375 \mathrm{~K}$.

${ }^{d}$ We cannot ascertain whether phases IIIa and IIIb and II are distinct phases or a single phase with thermal transformations, but have adopted a "phase" notation. (Compare reference 16.) 
as well as fusion near $582 \mathrm{~K}^{(12)}$ are in reasonable accord with the literature.

A complete description of the methods used and the transition temperatures therefrom can be found elsewhere. ${ }^{(10)}$ Neither the purity of their samples nor their heat-capacity values are provided in any of the references quoted. Comparison of the excess enthalpies and entropies is difficult as the method of drawing lattice curves affects the calculated excess values and the lattice curves can be drawn only when heat-capacity morphology is known precisely. This may account for the lack of enthalpies of transition ${ }^{(11)}$ and the complete absence of entropies of transitions.

Only three phase transformations-monoclinic (IIIa or IIIb)-to-monoclinic (III)to-orthorhombic (I)-to-isotropic liquid-have been considered probable in potassium acetate as the other transformations appear to be non-isothermal and apparently do not involve phase changes.

Hatibarua and Parry ${ }^{(13)}$ and Hatibarua ${ }^{(15)}$ studied the transitions in $\mathrm{K} 2 \mathrm{C}$ by crystallographic and thermal-expansion methods, respectively. The former investigators grew single crystals of the salt from the melt using dried polycrystalline commercial materials. They reported that $\mathrm{K} 2 \mathrm{C}$ has three polymorphs; the form existing from room temperature to a temperature of about $348 \mathrm{~K}$ was found to be monoclinic (Phase III) with either superlattice or an eight-fold increase in volume over the monoclinic polymorph between 348 and $428 \mathrm{~K}$ (Phase II); that above $428 \mathrm{~K}$ was observed to be orthorhombic (Phase I).

Hatibarua $^{(15)}$ found the transition from Phase I to Phase II (i.e. orthorhombic to monoclinic) to be characterized by a change in the structural symmetry and termination of the rapid expansion in the cell constant $a^{\prime}$. From the structural studies, he observed that the unit cell of Phase I consists of ionic double layers parallel to (100) of potassium and carboxylate ions firmly bound by ionic forces. Sandwiched between such double layers are the methyl groups in contact and bonded by much weaker van der Waals forces. He also observed a void in the unit cell of Phase I extending through the entire crystal along (100) and suggested that these two peculiarities seem to account for the thermal behavior of $\mathrm{K} 2 \mathrm{C}$.

Hatibarua, ${ }^{(16)}$ using crystallographic methods, found that as the transition temperature $428 \mathrm{~K}$ is approached, the acetate ions begin to librate between two equivalent sites. As a result, twin orientations of the low-temperature monoclinic phase occur. As the temperature increases, increasingly more acetate ions switch between the two sites until both orientations have statistically equal populations. The reflections corresponding to the twin orientations move towards each other as the temperature increases and coalesce at $428 \mathrm{~K}$. A dielectric study by the same author revealed no evidence for a ferro-to-para-electric transition $(503 \mathrm{~K})$ as hinted by Hazlewood et al., ${ }^{(12)}$ who suggested that Phase III could be a modulated structure obtained from the Phase II structure.

From our calorimetric studies, we found scant thermodynamic evidence for the existence of Phase III as the hump between 300 and $375 \mathrm{~K}$ and the bifurcated transition seem not to be phase transitions.

Jenkins and $O^{\prime} B^{\prime}$ rien $^{(14)}$ found the entropy change associated with the transition at $349 \mathrm{~K}$ (onset) to be of the order $R \cdot \ln 2$ and attributed this transition to orientational (order-disorder) effects. They used d.s.c., d.t.a., and Raman spectroscopy, and found 
only two transitions; the one at $349 \mathrm{~K}$ (onset) presumably corresponding to ours at 375 and the other at $413 \mathrm{~K}$ (onset) corresponding to our transition at $474 \mathrm{~K}$. Despite its prominence, the peak at $474 \mathrm{~K}$ has seldom been unambiguously identified as being within $85 \mathrm{~K}$ of our peak temperature. ${ }^{(14,17,18)}$

Thermal transformations were not detected in rubidium and cesium acetates between room temperature and the melting temperature. ${ }^{(13)}$ Two transitions were observed between 100 and $200 \mathrm{~K}$ in potassium butanoate, $\mathrm{K} 4 \mathrm{C},{ }^{(1)}$ whereas in potassium acetate a similar pair appear at much higher temperature ( 375 to $500 \mathrm{~K}$ ). Upon increasing the temperature, the longer hydrocarbon tail in $\mathrm{K} 4 \mathrm{C}$ may possibly acquire freedom at lower temperatures than does the much shorter tail in $\mathrm{K} 2 \mathrm{C}$.

Sodium acetate exhibits a transition at $21 \mathrm{~K},{ }^{(5)}$ while $\mathrm{K} 2 \mathrm{C}$ and $\mathrm{Li} 2 \mathrm{C}^{(19)}$ do not show a similar transition. The heat-capacity curves lie in the expected order. As already noted ${ }^{(13)}$ sodium and potassium acetates are not isostructural near $300 \mathrm{~K}$. The presence of the $21 \mathrm{~K}$ transition in sodium acetate ${ }^{(5)}$ was held responsible for the overall larger heat capacity of this salt compared to that of sodium propanoate but the nature of the transition is still unknown. After studying several compounds of lithium and potassium ${ }^{(4)}$ by the same method, we found that the odd-even effect observed in compounds with hydrocarbon tails at ambient temperatures is also a low-temperature phenomenon and that the heat-capacity curves increase above the transition monotonically in the $N_{C}$ sequence $6 \rightarrow 4 \rightarrow 3 \rightarrow 5 \rightarrow 7$.

We thank Professor T. R. Williams of the College of Wooster for the use of his d.s.c. apparatus.

\section{REFERENCES}

1. Westrum, E. F., Jr.; Ngeyi, S. P.; Franzosini, P. J. Chem. Thermodynamics 1987, $219,113$.

2. Ferloni, P.; Sanesi, M.; Franzosini, P. Z. Naturforsch. 1975, 30a, 1447.

3. Skoda, W. Kolloid-Z. Polym. 1969, 234, 1128.

4. Ngeyi, S. P. Ph.D. Thesis, Thermodynamic Properties of Lithium, Potassium, and Thallium(I) Alkanoates, The Universily of Michigan, Ann Arbor, MI, 1985.

5. Franzosini, P.; Plautz, W. A.; Westrum , E. F., Jr. J. Chem. Thermodynamics 1983, 15, 445.

6. Blaine, R. L. Thermal Analysis. Dupont, Analytical Instruments Division: Delaware.

7. Wendlandt, W. Thermal Methods of Analysis. Wiley: New York. 1974.

8. Westrum, E. F., Jr. Proceedings of NATO Advanced Study, Institute on Thermochemistry at Viana do Castello, Portugal. Ribeiro da Silva, M. A. V.: editor. Reidel: New York. 1984, p. 745.

9. Instructions for the Specific Heat Software Kit, Perkin Elmer, Norwalk, CT, 1983.

10. Franzosini, P.; Sanesi, M. Thermodynamic and Transport Properties of Organic Salts. Pergamon: New York. 1980.

11. Sokolov, N. M. Tezisy Dokl. X Nauch. Konf. S-M 1. 1956.

12. Hazlewood, F. J.; Rhodes, E.; Ubbelohde, A. R. Trans. Faraday Soc. 1966, 62, 3101.

13. Hatibarua, J.; Parry, G. S. Acta Cryst. 1972, B28, 3099.

14. Jenkins, T. E.: O'Brien, P. J. Phys. Chem. Solids 1983, 44, 565.

15. Hatibarua, J. Current Science 1978, 47, 719.

16. Hatibarua, J. Ph.D. Thesis, London University, 1968.

17. Diogenov, G. G.; Erlykov, A. N. Nauchn. Dokl. Vysshej Shkoly Khim. i Khim. Technol. 1958, 3, 413.

18. Diogenov, G. G.; Nurminsk. N. N.; Gimelshtein, V. G. Russ. J. Inorg. Chem. 1957, 2, 237.

19. Ferloni, P.; Kenesey, C. K.; Westrum, E. F., Jr. Personal communication, 1989. 